\title{
Programas de integridad en El Mercado de CAPITAles ARGENTINO, ESTADO DE SITUACIÓN AL 2020
}

\author{
INTEGRITY PROGRAMS IN THE ARGENTINE CAPITAL MARKET: \\ STATUS OF SITUATION AS OF 2020
}

María Marta Panario Centeno ${ }^{1}$ Paula Alejandra D’Onofrio ${ }^{2}$

https://doi.org/10.52292/j.eca.2021.2266

Fecha de recepción: 12/11/2020

Fecha de aceptación: 15/09/2021

\section{Resumen}

El presente trabajo tiene como objetivo conocer la situación actual en la implementación de programas de integridad, códigos de ética y líneas de denuncia y transparencia en las empresas del mercado de capitales argentino. Para ello se realizó un estudio descriptivo y cuantitativo de la información contenida en las páginas web institucionales de 200 empresas. Los datos que surgen del relevamiento efectuado muestran que la divulgación de programas de integridad y políticas anticorrupción es baja (22\%) y que existe mayor información sobre sus códigos de ética y conducta $(49,5 \%)$ y líneas de denuncia $(28 \%)$. Considerando que resulta esencial para las partes interesadas tener información completa e íntegra, entendemos que es a partir de la página web de cada entidad la vía con mayores posibilidades de difusión. Los datos arrojados por

1 Facultad de Ciencias Económicas. Universidad Católica Argentina. E-mail: maria_panario@ uca.edu.ar, panariocentenomaria@gmail.com. ORCID: https://orcid.org/0000-0002-3917-8549.

2 Facultad de Ciencias Económicas. Universidad de Buenos Aires. E-mail: pdonofrio1@gmail. com.ORCID: https:/ / orcid.org/0000-0001-6483-0087. 
el relevamiento evidencian que en Argentina es necesario elevar los niveles de transparencia de la información en las entidades cotizadas.

Palabras clave: programas de integridad, códigos de ética, líneas de transparencia, mercado de capitales, Argentina.

\begin{abstract}
The objective of this work is to know the current situation in the implementation of integrity programs, codes of ethics and reporting and transparency lines in companies in the Argentine capital market. For this, a descriptive and quantitative study of the information contained in the institutional websites of 200 companies was carried out. The data arising from the survey carried out shows that the disclosure of integrity programs and anti-corruption policies is low $(22 \%)$ and that there is more information on their codes of ethics and conduct $(49.5 \%)$ and hotlines (28\%). Considering that it is essential for interested parties to have complete and complete information, we understand that it is from the website of each entity the route with the greatest possibilities of dissemination. The data produced by the survey show that in Argentina it is necessary to increase the levels of transparency of information in listed entities.
\end{abstract}

Keywords: Integrity programs, codes of ethics, transparency lines, capital markets, Argentina.

JEL: M10, M4, M14. 
Programas de integridad en el mercado de capitales argentino...

https://doi.org/10.52292/j.eca.2021.2266

\section{Introducción}

El presente trabajo tiene como objetivo conocer la situación actual en la implementación de programas de integridad, códigos de ética y líneas de denuncia y transparencia sugeridos por la Ley de Responsabilidad Penal de las Personas Jurídicas, en las empresas del mercado de capitales argentino ${ }^{3}$.

El 1 de diciembre de 2017 se publicó la ley mencionada en el párrafo anterior, bajo el número 27401 (2017), como una de las políticas de alineación con la Organización para la Cooperación y el Desarrollo Económico (OCDE), que luego fue reglamentada por el decreto número 277/18 (2018) del 6 de abril de ese año. En esta misma línea, la Comisión Nacional de Valores (CNV) dictó la Resolución 797 (2019) con el objeto de mejorar la calidad de la información hacia terceros en materia de gobierno corporativo y sustentabilidad. La misma fue publicada el 14/06/2019 y afecta a las entidades que se encuentren en el régimen de oferta pública de sus valores negociables, y las que soliciten autorización para ingresar al régimen de oferta pública.

Los primeros estudios de gobierno corporativo datan de la década del 90. En los inicios, se focalizaron en cuestiones de agencia, composición del directorio y remuneraciones; luego, con el correr del tiempo, fueron incorporándose temáticas de control de riesgo y transparencia. En un principio estaban destinados, principalmente, a mejorar el clima de inversión (The Institute of Chartered Accountants in England and Wales [ICAEW], 1992). Si bien esto se mantiene hasta nuestros días, las primeras recomendaciones de OCDE en el año 1999 se alinearon a las necesidades de los grupos de interés en general y apuntaron a la transparencia e integridad (Organización para la Cooperación y el Desarrollo Económicos [OCDE], 2016).

Desde entonces y hasta el presente las temáticas de gobierno corporativo fueron transformándose en buenas prácticas de aplicación general a través de los principios de Pacto Global de Naciones Unidas (Pacto Global de Naciones Unidas [PG], 2018) y las iniciativas del Global Reporting Initiative (https:// www.globalreporting.org/), entre otras propuestas voluntarias.

Sin embargo, a pesar de generar informes con mayor transparencia, los

3 El presente trabajo se enmarca en el proyecto de Desarrollo Estratégico (PDE) de la Universidad de Buenos Aires. PDE_9_2019 “Corrupción, fraude empresario y sistemas de cumplimiento en las organizaciones". Este tiene por fin el desarrollo de herramientas del sistema de información que permitan establecer el correcto funcionamiento de los sistemas de cumplimiento en las organizaciones. 
fraudes en las organizaciones se mantienen activos. Esto motivó que varios países fueran generando legislación relativa a las ventajas de tener programas de autorregulación y control para evitar situaciones de corrupción. En D’Onofrio (2020), se analizó la legislación de Francia, España, Reino Unido, Estados Unidos y algunos países de América Latina con el objetivo de detectar cómo se incentiva a las organizaciones a desarrollar programas de integridad.

En Argentina, la publicación de la Ley 27401 (2017) favoreció el proceso de actualización o implementación de programas de integridad en las organizaciones. Del mismo modo, la inserción de esta favoreció la modificación del código de gobierno corporativo propuesto por la Comisión Nacional de Valores alineándolo con las políticas de la mencionada legislación.

En esta línea, para el desarrollo del presente trabajo, se realizó un relevamiento descriptivo y cuantitativo de la información contenida en las páginas web institucionales de 200 empresas emisoras. Dicho estado de situación adquiere relevancia en los principios de transparencia y rendición de cuentas de las políticas, programas y procedimientos de integridad y buen gobierno en empresas de todo tipo. La transparencia implica la publicación de aquella información que permita a distintos actores (medios de comunicación, gobiernos, inversores, clientes) monitorear la gestión organizacional.

A continuación, en la sección 2, el trabajo presenta una primera parte de antecedentes normativos, los cuales justifican el interés de la investigación posterior. En primer lugar, se analiza la Ley 27401 (2017) de Responsabilidad Penal de las Personas Jurídicas, como marco de los programas de integridad. Dentro de estos procedimientos de autorregulación se estudian las características principales relativas a programas de integridad, códigos de ética y líneas de denuncia y transparencia. A partir del dictado de esta ley, los códigos de gobierno societario previstos para las empresas que hacen oferta pública de sus acciones en la Comisión Nacional de Valores sufren cambios resaltando temas como la integridad y la ética, por ello se analiza la regulación referida al particular (Resolución 797, 2019). La segunda parte del trabajo se centra en el desarrollo metodológico y análisis de las empresas mencionadas en apartados anteriores (sección 3). Por último, se presentan resultados (sección 4) y conclusiones del trabajo (sección 5). 


\section{Marco de antecedentes}

\subsection{La Ley Penal de Responsabilidad de Personas Jurídicas}

La Ley 27401 (2017) sobre la Responsabilidad Penal de las Personas Jurídicas obedeció a la necesidad de que la Argentina cumpliera con los requisitos para ingresar como Estado miembro a la OCDE, y a compromisos y convenciones internacionales. La norma establece un régimen de responsabilidad penal aplicable a las personas jurídicas privadas que sean de capital nacional o extranjero, pudiendo o no tener participación estatal en su capital que cometan alguno de los siguientes delitos:

- delitos de cohecho y tráfico de influencias (Código Penal de la Nación Argentina [CPNA], 1921, artículos 258 y 258 bis; Ley 11179, 1984 y actualizaciones);

- negociaciones incompatibles con el ejercicio de las funciones públicas (CPNA, 1921, artículo 265; Ley 11179, 1984 y actualizaciones);

- concusión (CPNA, 1921, artículo 268; Ley 11179, 1984 y actualizaciones);

- enriquecimiento ilícito de funcionarios y empleados (CPNA, 1921, artículos 268.1 y 268.2; Ley 11179, 1984 y actualizaciones); y

- balances e informes falsos agravados (CPNA, 1921, artículo 300 bis; Ley 11179, 1984 y actualizaciones).

Estos delitos pueden ser cometidos, según se menciona en su artículo 2, de forma directa o indirecta: a) con su intervención o b) en su nombre, interés o beneficio (Ley 27401, 2017) y serán según el artículo 7 pasibles de diversas penalidades, entre las cuales podemos mencionar: multas; suspensión total o parcial de actividades, suspensión para participar en concursos o licitaciones estatales de obras o servicios públicos o en cualquier otra actividad vinculada con el Estado; disolución y liquidación de la personería; pérdida o suspensión de los beneficios estatales que tuviere, entre otros.

La ley fomenta la cooperación público-privada para promover la autorregulación empresarial. Su objetivo es generar incentivos para que las personas jurídicas prevengan la comisión de ciertos delitos mediante la implementación de programas de integridad descriptos en su artículo 23, los cuales según el artículo 9 son atenuantes de las penas y responsabilidades antes mencionadas. 


\subsubsection{Los programas de integridad}

La legislación establece la implementación de los programas de integridad y, además, instruye sobre los contenidos mínimos que deben contener y ejecuta sugerencias de contenido. Define a los programas de integridad, en su art. 22, como el "conjunto de acciones, mecanismos y procedimientos internos de promoción de la integridad, supervisión y control, orientados a prevenir, detectar y corregir irregularidades y actos ilícitos" (Ley 27401, 2017). De la misma manera, se exige que el programa guarde "relación con los riesgos propios de la actividad que la persona jurídica realiza, su dimensión y capacidad económica". Se coincide plenamente con esta norma, debido a que los programas de integridad no pueden ser estandarizados, es necesario diseñarlos "a medida" de cada organización, ya que no existen entes idénticos.

- La norma determina que el programa deberá contener, "al menos", los siguientes elementos:

- Un código de ética o de conducta, o la existencia de políticas y procedimientos de integridad aplicables a todos los directores, administradores y empleados, independientemente del cargo o función ejercidos, que guíen la planificación y ejecución de sus tareas para prevenir la comisión de los delitos bajo la Ley 27401 (2017).

- Reglas y procedimientos específicos para prevenir ilícitos en el ámbito de concursos y procesos licitatorios, en la ejecución de contratos administrativos o en cualquier otra interacción con el sector público.

Capacitaciones periódicas sobre el programa de compliance a directores, administradores y empleados (Ley 27401, 2017, art. 23). Adicionalmente, la ley establece que el programa "podrá" contener los siguientes elementos:

- Análisis periódico de riesgos y la consecuente adaptación del programa de integridad.

- Apoyo visible e inequívoco al programa de integridad por parte de la alta dirección y gerencia.

- Canales internos de denuncia de irregularidades, abiertos a terceros y adecuadamente difundidos.

- Una política de protección de denunciantes contra represalias.

- Un sistema de investigación interna que respete los derechos de los investigados e imponga sanciones efectivas a las violaciones del código de ética o conducta. 
- Procedimientos que comprueben la integridad y trayectoria de terceros o socios de negocios, incluyendo proveedores, distribuidores, prestadores de servicios, agentes e intermediarios, al momento de contratar sus servicios durante la relación comercial.

- La debida diligencia durante los procesos de transformación societaria y adquisiciones, para la verificación de irregularidades, de hechos ilícitos o de la existencia de vulnerabilidades en las personas jurídicas involucradas.

- El monitoreo y evaluación continua de la efectividad del programa de integridad.

- Un responsable interno a cargo del desarrollo, coordinación y supervisión del programa de integridad.

- El cumplimiento de las exigencias reglamentarias que sobre estos programas dicten las respectivas autoridades del poder de policía nacional, provincial, municipal o comunal que rija la actividad de la persona jurídica (Ley 27401, 2017, art. 23).

Más allá del contenido que la norma establece, y de las ventajas que otorga a quienes lo posean, creemos que estos programas de autocontrol son un mecanismo imprescindible para anticiparnos y prevenir los riesgos a los cuáles se enfrenta una organización.

\subsubsection{Código de ética y conducta}

Uno de los elementos claves de los programas de integridad lo constituye el código de ética y conducta, que transmite el marco legal asociado a la organización y los valores adoptados por la alta dirección. El código de ética de una organización es un documento que reúne los principios y valores que son reconocidos y respetados dentro de la misma. Debe transmitir los valores éticos empresariales no solo internamente, sino también a los socios en los negocios. Algunos son internos y externos, pero muchas organizaciones tienen códigos separados porque los riesgos son distintos.

Cada organización debería definir su contenido de acuerdo con las características propias del sector en el que opera y sus riesgos intrínsecos. Además, debe preverse la obligatoriedad del código y su aplicación a todos los integrantes de la empresa. También debe ser aprobado por las máximas autoridades de la organización, revisado y actualizado.

En cuanto a su contenido, la Oficina Anticorrupción (OA) y el Programa de las Naciones Unidas para el Desarrollo (PNUD) plantean que debe ser comunicado 
en un lenguaje simple y claro, debe tener una lectura amena y accesible para todos los integrantes del ente, de la misma manera que debe dejar en claro las sanciones previstas en caso de incumplimiento. Dentro del mismo, es importante que contengan reglas para la gestión de los conflictos de intereses y un régimen de obsequios. Además, es fundamental que se incluya la obligación de reportar internamente cualquier infracción al Código y los canales para hacerlo, los cuales serán abordados en el apartado siguiente (Oficina Anticorrupción [OA], 2019a, 2019b; Programa de Naciones Unidas para el Desarrollo [PNUD], 2016).

El código debe ser comunicado a todos los miembros según su alcance, debiendo los mismos manifestar su compromiso, comprensión y adhesión. El alcance del código puede incluir a los terceros con quienes la entidad interactúa. Es muy importante que los valores organizacionales sean transmitidos a toda la cadena de valor con el fin de limitar aún más los riesgos.

Si bien no es objeto de la presente investigación, para asegurar la vigencia de estos es necesario que se sometan a actualizaciones periódicas y que las mismas sean acompañadas de una capacitación adecuada. Destaca Preziosa (2018, citada por Durrieu y Saccani, 2018) que "es necesario que la capacitación tenga un espacio para que los sujetos que participan de ella puedan reflexionar y dialogar. Es decir, que no solo reciban una instrucción unidireccional sobre las normas y las consecuencias de su incumplimiento" (p. 231).

\subsubsection{Líneas de denuncia y líneas de transparencia}

La implementación de canales para la recepción de denuncias e instancias de investigación y sanción internas también son parte de las recomendaciones en materia de integridad tanto a nivel internacional como nacional. La mayoría de los delitos contemplados en estos programas tienen la particularidad de ser cometidos en privado, por lo que en muchos casos la única manera de detectarlos es mediante la denuncia de un tercero o de alguien afectado, pero utilizando canales que aseguren la confidencialidad. Según un reciente informe de 2020 de la Asociación de Examinadores de Fraude Certificados (ACFE), se indica que el 49 \% de los fraudes de la región se detectan a través de información provista por empleados o terceros, aumentando significativamente su detección cuando se establecen canales de denuncia.

Comúnmente conocido como whistleblowing, las líneas de denuncias son canales que, de estar bien implementados, están al alcance de empleados, proveedores, trabajadores tercerizados, organismos de control e, incluso, del público 
en general, para exteriorizar prácticas ilegales, inmorales y/o ilegítimas que son cometidas por empleados de una organización hacia otras personas u organizaciones que son denunciadas por miembros de la organización (D'Onofrio, 2018). Asimismo, debe existir una investigación exhaustiva que compruebe que dichas denuncias fueron consumadas sobre hechos reales, método esencial para la detección de fraudes y conductas irregulares en las empresas.

Los canales de denuncia pueden ser destinados a los empleados de la organización o bien extenderse a la cadena de valor. La administración de estos puede ser realizada internamente, siempre que se asegure la confidencialidad de los datos del denunciante y se lo proteja, garantizando que la denuncia es recibida de manera neutral.

Las denuncias tempranas pueden dar lugar a corregir situaciones o al menos prevenir sus consecuencias. La exposición pública puede entenderse como una responsabilidad hacia la sociedad en su conjunto, un acto de lealtad hacia la sociedad, pero desde la perspectiva de la organización puede ser tomado como una traición. La lealtad de los miembros de una organización debe ser ejercida con el cumplimiento normativo, el código de ética y conducta, la misión y los valores, y no con las personas que lo promueven.

\subsection{El Código de gobierno societario y los programas de integridad}

Antes de comenzar a hablar del código de gobierno societario, resulta necesario precisar el concepto de "Corporate Governance". Tal como menciona Apreda (2007), desde la década del 70 varios aportes doctrinarios trataron de dar una definición precisa, objetivo bastante complejo, porque a medida que se profundiza se fueron incorporando nuevas facetas. Así como en un principio se centró en las tensiones internas primero entre administradores y accionistas, luego se fueron abordando conflictos entre los mismos accionistas, los empleados, los reguladores, para culminar a partir de la valorización de la responsabilidad social empresaria con los conflictos entre los intereses de la "comunidad en general, las sociedades y sus protagonistas" (Salvochea, 2016, p. 5).

En Argentina las sociedades sujetas a la regulación de la CNV se encuentran obligadas a la presentación de un Código de Gobierno Societario conforme a la Resolución 797 (2019). Alineándose con la Ley de Responsabilidad Penal de las Personas Jurídicas 27401 (2017), esta resolución presenta en su inciso F) Ética, integridad y cumplimiento, los siguientes principios: 
- XVII. El Directorio debe diseñar y establecer estructuras y prácticas apropiadas para promover una cultura de ética, integridad y cumplimiento de normas que prevenga, detecte y aborde faltas corporativas o personales serias.

- XVIII. El Directorio asegurará el establecimiento de mecanismos formales para prevenir y en su defecto lidiar con los conflictos de interés que puedan surgir en la administración y dirección de la compañía. Deberá contar con procedimientos formales que busquen asegurar que las transacciones entre partes relacionadas se realicen en miras del mejor interés de la compañía y el tratamiento equitativo de todos sus accionistas (Resolución 797, 2019).

- En el mencionado inciso F) se establece que el Directorio debe aprobar un Código de Ética y Conducta que refleje los valores y principios éticos y de integridad, así como también la cultura de la compañía. Este debe ser comunicado y tener como alcance a todos los directores, gerentes y empleados de la compañía. También hace referencia a un programa de integridad, en base a los riesgos, dimensión y capacidad económica del ente. El programa dispone dentro del apartado 23 del mencionado inciso, lo siguiente:

(i) capacitaciones periódicas a directores, administradores y empleados sobre temas de ética, integridad y cumplimiento; (ii) canales internos de denuncia de irregularidades, abiertos a terceros y adecuadamente difundidos; (iii) una política de protección de denunciantes contra represalias; y un sistema de investigación interna que respete los derechos de los investigados e imponga sanciones efectivas a las violaciones del Código de Ética y Conducta; (iv) políticas de integridad en procedimientos licitatorios; (v) mecanismos para análisis periódico de riesgos, monitoreo y evaluación del Programa; y (vi) procedimientos que comprueben la integridad y trayectoria de terceros o socios de negocios (incluyendo la debida diligencia para la verificación de irregularidades, de hechos ilícitos o de la existencia de vulnerabilidades durante los procesos de transformación societaria y adquisiciones), incluyendo proveedores, distribuidores, prestadores de servicios, agentes e intermediarios (Resolución 797, 2019).

Claramente adopta el modelo conocido como tone from the top, donde la dirección debe "marcar el tono" que refleje el compromiso con los valores organizacionales, dándolo a conocer de manera clara a los miembros de la organización y su cadena de valor.

\section{Metodología}

El relevamiento tiene como objetivo conocer la situación actual en la implementación de "programas de integridad", "códigos de ética" y "líneas de denuncia 
o transparencia" en las empresas del mercado de capitales argentino. La muestra objeto de investigación está compuesta por 200 empresas listadas como emisoras en la CNV al 2017. La investigación es descriptiva porque busca presentar la situación actual de la adopción de programas de integridad, códigos de ética y líneas de denuncia en el mercado de valores argentino, limitándose únicamente a su medición y descripción sin alterar o manipular ninguna de las variables a investigar, las cuales tienen que ver con el perfil económico de las empresas y la presencia de programas de integridad, códigos de ética y líneas de denuncia en las páginas web institucionales de las empresas.

La recolección y el análisis de los datos se han llevado a cabo entre los meses de abril y julio de 2020 a través de información disponible en las páginas web institucionales de las empresas. Para clasificar a las empresas por sector económico se tuvo en cuenta el Clasificador Nacional de Actividades Económicas 2010 del Instituto Nacional de Estadísticas y Censos (Instituto Nacional de Estadística y Censos [INDEC], 2010), considerando para su clasificación la actividad principal mencionada en la ficha técnica Bolsar de la Bolsa de Comercio de Buenos Aires. En cuanto a la información sobre la presencia de programas de integridad, código de éticas y líneas de denuncia en los sitios webs institucionales de las empresas, las misma se midieron de manera binaria (presenta o no presenta). El criterio para considerar que presenta ha sido si la empresa lo menciona. La búsqueda en el sitio web se realizó recorriendo el mismo visualmente, observando en una primera instancia la sección institucional, de gobierno corporativo o relación con los accionistas, además, para los casos que no se encontraron mención a las variables en esas secciones, se ha utilizado la herramienta 'Buscar' del navegador con determinadas palabras clave, entre las cuales podemos mencionar: programa de integridad, políticas anticorrupción, departamento de compliance, líneas de denuncias, líneas éticas, líneas de transparencia, código de ética y conducta. Los datos se han procesado con Excel realizándose un análisis descriptivo y bivariado relacionando el sector económico con la presencia de programas de integridad, códigos de ética y líneas de transparencia. De esta manera, se contestaron los siguientes interrogantes:

- ¿La empresa manifiesta tener en su web programas de integridad, políticas anticorrupción, departamento de compliance?

- ¿Se visualizan líneas de denuncia, líneas éticas y/o líneas de transparencia?

- ¿La empresa divulga a los distintos stakeholders un código de ética y conducta? 


\section{Resultados}

Los datos del relevamiento están disponibles para su consulta en el apéndice I en línea al que puede accederse en este enlace: bit.ly/3EhIrTg. A continuación, se sintetizan los principales resultados obtenidos.

\subsection{Caracterización de la muestra}

En cuanto al perfil sectorial de las empresas analizadas podemos mencionar que los sectores más predominantes dentro del mercado de capitales argentino, como se puede observar en la figura 1 que a continuación se muestra, son: las entidades financieras; la industria manufacturera, que incluye empresas que van desde la elaboración de productos alimenticios hasta productos químicos, farmacéuticos y electrónicos; las empresas de suministro de electricidad, gas, vapor y aire acondicionado; explotación de minas y canteras que incluye a empresas de extracción de petróleo y gas natural, y las empresas constructoras.

Figura 1. Perfil sectorial de las empresas analizadas

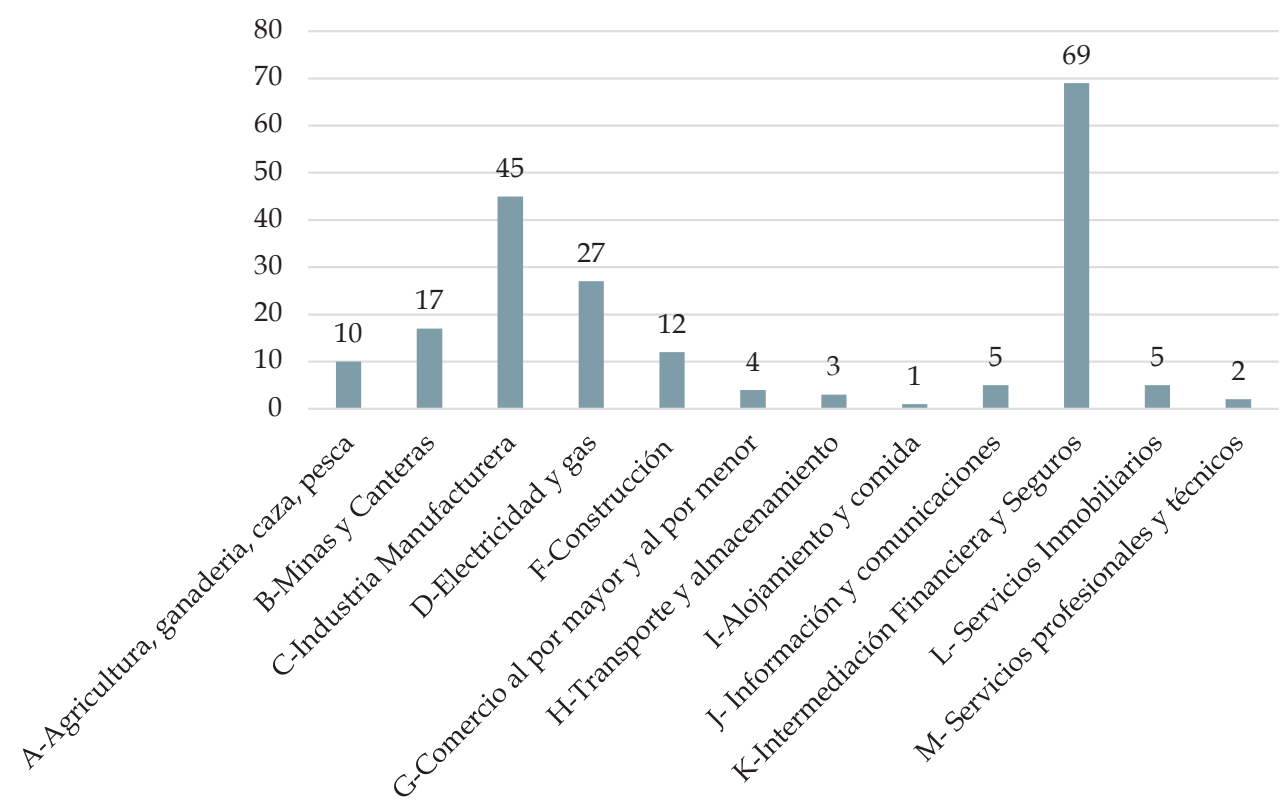

Fuente: elaboración propia sobre la base de datos obtenidos del apéndice I. 


\subsection{Análisis descriptivo}

Los programas de integridad y las políticas anticorrupción juegan un papel central para que la persona jurídica quede eximida y/o se reduzca el alcance de las penas previstas por los delitos que se encuentran contemplados en la Ley 27401 (2017). En la figura 2 se puede observar que son muy pocas las empresas que mencionan tener programas de integridad y políticas anticorrupción en sus páginas web. De las 200 empresas analizadas, solamente el $22 \%$ divulga y pone a disposición del público sus políticas, pronunciamientos y compromisos de integridad, mientras que más del $60 \%$ aún no lo hace.

En cuanto a los canales de denuncia y los códigos de ética, los datos relevados muestran que el 49,5 \% de las compañías manifiestan contar con códigos de ética y conducta como un elemento esencial y uno de los tres que obligatoriamente debe incluir un programa de integridad de acuerdo a los términos de la Ley 27401 (2017), mientras que solo el 28 \% de las compañías utilizan canales de denuncias para reportar fraudes, hechos de corrupción, delitos informáticos y cualquier otro comportamiento no ético o irregular. También se puede observar que del total de empresas relevadas solamente el $21 \%$ (37 empresas) presentan programas de integridad, tienen códigos de éticas y líneas de denuncias, en cambio el 42 \% (75 empresas) no tiene ninguna de las tres variables.

Figura 2. Presencia de programas de integridad, códigos de ética y líneas de transparencia en la web de las empresas del mercado de capitales argentino

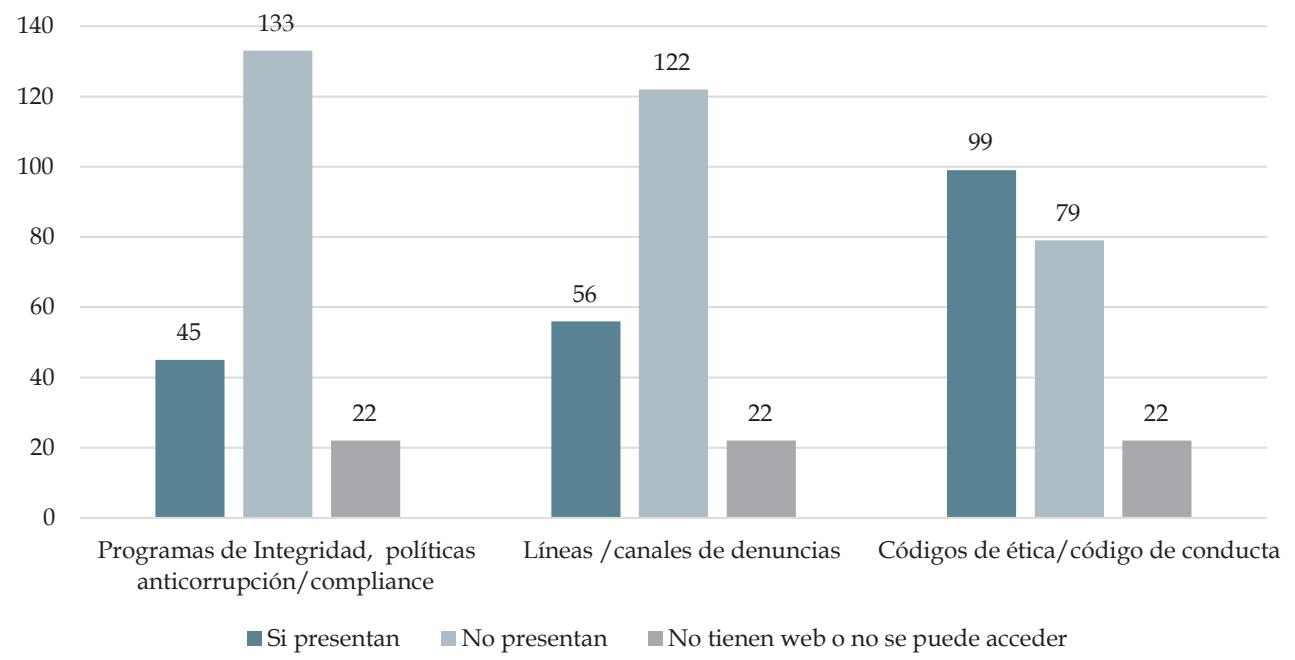

Fuente: elaboración propia sobre la base de datos obtenidos del apéndice I. 


\subsection{Análisis bivariado}

En cuanto a la implementación de programas de integridad y políticas anticorrupción, se puede mencionar, como se muestra en la figura 3, que los mismos se encuentran mayoritariamente presentes dentro del sector electricidad, gas, vapor y aire acondicionado, con un $33 \%$, la industria manufacturera (16 \%), las constructoras (11\%) y las entidades financieras (11\%). Sin embargo, la implementación de programas de integridad es nula en las empresas del sector agropecuario, ganadería y pesca, en el sector de alojamiento y hotelería y dentro de las empresas que brindan servicios inmobiliarios.

Figura 3. Presencia de programas de integridad y políticas anticorrupción por sector económico

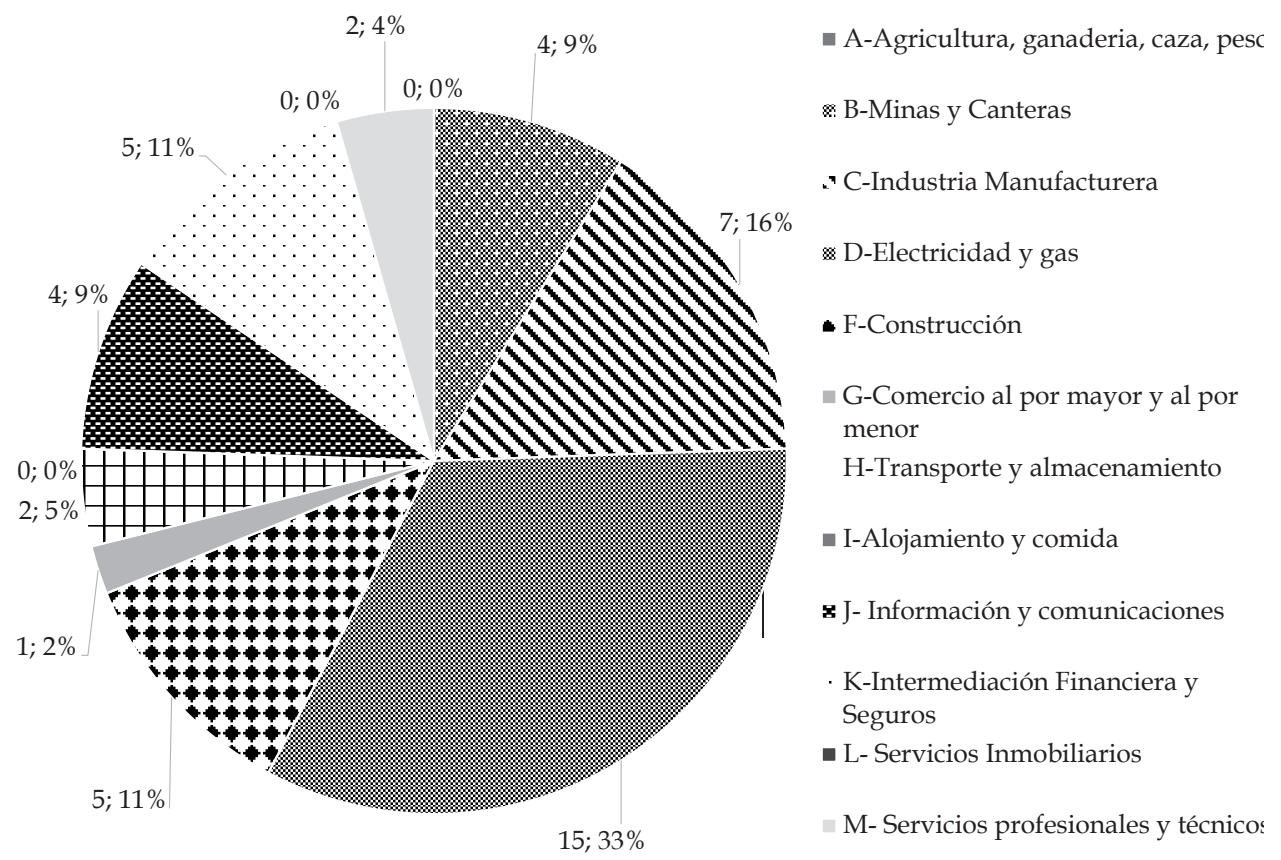

Fuente: elaboración propia sobre la base de datos obtenidos del apéndice I.

Por su parte, los códigos de ética y conducta se encuentran presentes mayoritariamente en las entidades financieras y de seguros (34\%), seguidas de las empresas de suministros de electricidad, gas, vapor y aire acondicionado $(21 \%)$ y la industria manufacturera (17\%), como se puede observar en la figura 4 . 
Programas de integridad en el mercado de capitales argentino... https://doi.org/10.52292/j.eca.2021.2266

Figura 4. Presencia de códigos de ética y conducta por sector económico

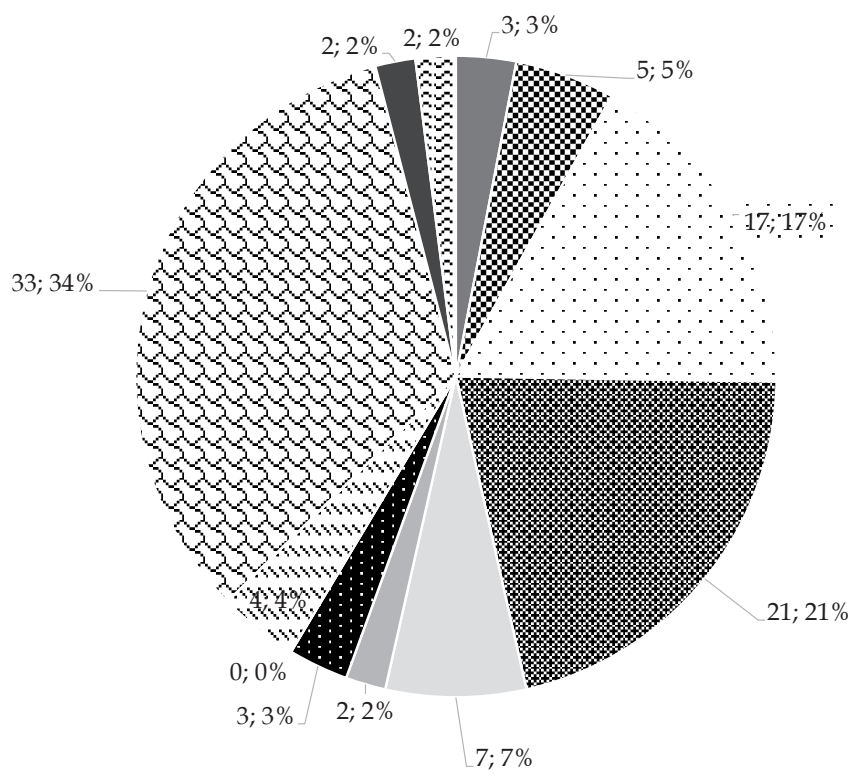

- A-Agricultura, ganaderia, caza, pesca

B-Minas y Canteras

- C-Industria Manufacturera

* D-Electricidad y gas

F-Construcción

- G-Comercio al por mayor y al por menor

- H-Transporte y

almacenamiento

- I-Alojamiento y comida

$\checkmark$ J- Información y comunicaciones

$\because$ K-Intermediación Financiera y Seguros

- L- Servicios Inmobiliarios

$\therefore$ M- Servicios profesionales y técnicos

Fuente: elaboración propia sobre la base de datos obtenidos del apéndice I.

Los canales de denuncia y líneas de transparencia que están al alcance de empleados, proveedores, trabajadores tercerizados, organismos de control e, incluso, del público en general se encuentran implementados, como se puede observar en la figura 5, en empresas de electricidad, gas, vapor y aire acondicionado (32\%), empresas manufactureras (20\%), entidades financieras (16 \%) y constructoras (11\%). 
Figura 5. Presencia de canales de denuncia y líneas ética por sector económico

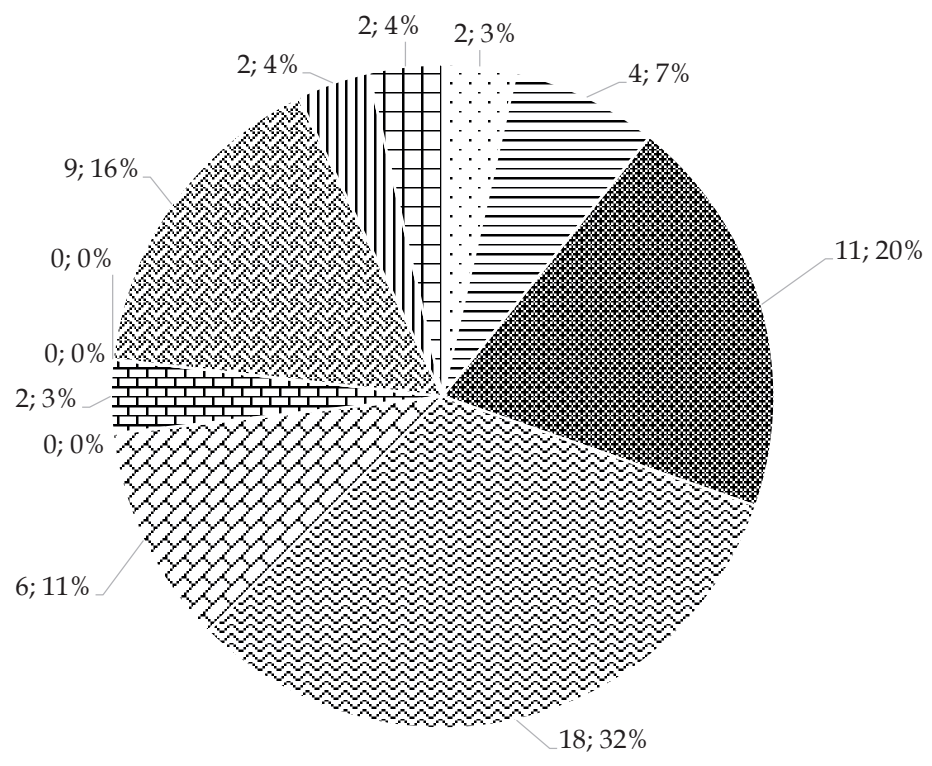
- A-Agricultura, ganaderia, caza, pesca
- B-Minas y Canteras
C-Industria Manufacturera
$\therefore$ D-Electricidad y gas
y F-Construcción
G-Comercio al por mayor $\mathrm{y}$ al por menor
ᄂ H-Transporte y almacenamiento
- I-Alojamiento y comida
· J- Información y comunicaciones
* K-Intermediación
Financiera y Seguros
II L- Servicios Inmobiliarios
I M- Servicios profesionales y técnicos

Fuente: elaboración propia sobre la base de datos obtenidos del apéndice I.

\section{Conclusiones}

A nivel internacional y nacional existe un creciente interés en promover el cumplimiento de estándares de Integridad y Buen Gobierno en las entidades cotizadas. La aplicación de políticas en este tipo de entidades es crucial por el alto impacto social y económico que generan en los mercados en los cuales operan.

En Argentina, la necesidad de desarrollar políticas de integridad en las organizaciones ha sido impulsada por la existencia de un nuevo marco normativo que responsabiliza penalmente a las personas jurídicas por un conjunto de delitos asociados a la corrupción (Ley 27401, 2017).

La Ley 27401 (2017) otorga a las empresas que desarrollen programas de integridad importantes incentivos durante las etapas del proceso judicial, y aun antes del mismo. En el caso de contrataciones con el Estado, resulta mandatorio la elaboración de estos programas de autorregulación empresarial. Pese a que la norma empezó a regir el 1 de marzo de 2018, todavía sigue teniendo un camino por recorrer. 
Los datos que surgen del relevamiento efectuado muestran que las empresas empiezan a interiorizarse de la nueva ley en forma muy paulatina, que la divulgación de programas de integridad y políticas anticorrupción en sus páginas web es baja, sin embargo, la mayor divulgación de sus códigos de ética nos daría un indicio de la existencia de programas o al menos procedimientos de integridad, ya que los mismos son componentes claves. Lo que resulta más preocupante es la falta de difusión masiva de las líneas de denuncia, ya que es el mecanismo más utilizado para descubrir los delitos alcanzados en la regulación según datos de la Association of Certified Fraud Examiner (2020).

Las entidades financieras y de seguros cuentan con regulación adicional que promueve la transparencia por ello no sorprende el buen nivel de información que presentan, sin embargo notamos que las empresas dedicadas al suministro de electricidad y gas, la industria manufacturera y las constructoras son también sectores económicos que informan en muy buen grado en sus páginas webs. En relación a esto último no tenemos otro argumento que el reconocimiento como valor agregado de la misma en la búsqueda de legitimidad y transparencia.

Resulta esencial que las partes interesadas y el público en general puedan informarse respecto de cuáles son las políticas, programas, procedimientos, valores, compromisos que hacen a la integridad y el buen gobierno societario. Una forma de hacerlo es a partir de la página web oficial de cada entidad. Los datos arrojados por el relevamiento evidencian que en Argentina es necesario elevar los niveles de transparencia en las entidades cotizadas.

En ese sentido, es importante que las políticas relacionadas con los valores organizacionales, la integridad, la anticorrupción y la transparencia sean divulgadas en la página web de la organización. Dichas políticas son instrumentos esenciales desde la perspectiva de la gobernanza ya que permiten guiar la planificación y ejecución de tareas o labores de forma tal de prevenir la comisión de los delitos de corrupción. Por ello, es esencial que se difunda en los canales de comunicación abierta que presenta la organización de forma que esté disponible para su consulta en todo momento.

\section{Referencias}

Apreda, R. (2007). Corporate Governance. La Ley.

Association of Certified Fraud Examiner (2020). 2020 Report to the Nations. Global Study on Occupational Fraud and Abuse. Recuperado de https:/ / acfepublic.s3-us-west-2.amazonaws.com/2020-Report-to-the-Nations.pdf 
Código Penal de la Nación Argentina [CPNA]. Artículos 258, 258bis, 265, 268, 268.1, 268.2 y 300. Septiembre de 1921 (Argentina).

Decreto 277 de 2018 [con fuerza de ley]. Reglamenta Ley Nro. 27.401 de Responsabilidad Penal. 5 de abril de 2018. B. O. No 22307.

D’Onofrio, P. A. (2018, octubre). Buenas Prácticas de Gobierno en Argentina [Ponencia]. XXXIX Jornadas Universitarias de Contabilidad \& VI Jornadas Universitarias Internacionales. Realizadas en la Universidad Nacional de Misiones, Misiones.

Durrieu, N. \& Saccani, R. R. (2018). Compliance, Anticorrupción y Responsabilidad Penal de las personas jurídicas. La Ley.

Institute of Chartered Accountants in England and Wales (1992). The Financial Aspects of Corporate Governance.

Instituto Nacional de Estadística y Censos (2010). Clasificador Nacional de Actividades Económicas. Notas Metodológicas.

Ley 27401 de 2017. Ley Responsabilidad Penal. 1 de diciembre de 2017.

Oficina Anticorrupción (2019a). Lineamientos para la implementación de Programas de Integridad Ley 27.401. Responsabilidad Penal de Personas Jurídicas. Ministerio de Justicia y Derechos Humanos Presidencia de la Nación. Recuperado de https://www.argentina.gob.ar/sites/default/files/lineamientos_para_la_ implementacion.pdf

Oficina Anticorrupción (2019b). Guía complementaria para la implementación de programas de integridad en PyMEs, Ministerio de Justicia y Derechos Humanos Presidencia de la Nación. Recuperado de https:/ / www.argentina.gob. ar/sites/default/files/guia_pymes.pdf

Organización para la Cooperación y el Desarrollo Económicos (2016). Principios de Gobierno Corporativo de la OCDE y del G20. París: Éditions OCDE. Recuperado de https:/ / www.oecd.org/daf/ca/corporategovernanceprinciples/37191543.pdf

Pacto Global de Naciones Unidas (26 de enero de 2018). Una llamada a la acción para empresas sostenibles https:/ / www.pactomundial.org/wp-content/uploads/2018/02/Flyer-New-Strategy-GC-2018_20180126.pdf

Programa de las Naciones Unidas para el Desarrollo (2016). Manual para la elaboración de un Código de Ética. Recuperado de https:/ / www.serviciocivil. cl/wp-content/uploads/2017/06/manual_elaboracion_codigo_etica_poretapa_24062016.pdf

Resolución 797 de 2019 [Comisión Nacional de Valores]. Por la cual se reforma el Código de Gobierno Societario. 19 de junio de 2019.

Salvochea, R (2016). Mercados y Buen Gobierno Corporativo. La Ley. 
(C) 2021 por los autores; licencia otorgada a la revista Escritos Contables y de Administración. Este artículo es de acceso abierto y distribuido bajo los términos y condiciones de una licencia Atribución-No Comercial 4.0 Internacional (CC BY-NC 4.0) de Creative Commons. Para ver una copia de esta licencia, visite https://creativecommons.org/licenses/by-nc/4.0/ 